\title{
Cost of Managing Type 2 Diabetes Before and After Initiating Dipeptidyl Peptidase 4 Inhibitor Treatment: A Longitudinal Study Using a French Public Health Insurance Database
}

\author{
Corinne Emery • Elodie Torreton · Sylvie Dejager • Laurie Levy-Bachelot • \\ Sébastien Bineau · Bruno Detournay (D)
}

Received: November 29, 2019 / Published online: January 17, 2020

(C) The Author(s) 2020

\begin{abstract}
Introduction: Diabetes is a growing epidemic that imposes a substantial economic burden on healthcare systems. This study aimed to evaluate the cost of managing type 2 diabetes (T2D) with dipeptidyl peptidase 4 inhibitors (DPP4Is) using real-world data.

Method: This longitudinal study used data from the French EGB (Echantillon Généraliste des Bénéficiaires) database. The annual average direct healthcare cost of treating patients with T2D was calculated 3 years prior and 3 years after initiation of DPP4I therapy. Actual total ambulatory and hospital care expenditure for the 3 years after DPP4I initiation was compared to projected costs. The distribution of costs
\end{abstract}

Enhanced Digital Features To view enhanced digital features for this article go to https://doi.org/10.6084/ m9.figshare.11523102.

Electronic Supplementary Material The online version of this article (https://doi.org/10.1007/s13300020-00760-x) contains supplementary material, which is available to authorized users.

C. Emery · E. Torreton · B. Detournay $(\varangle)$

Cemka, 43, boulevard Maréchal Joffre, 92340 Bourg

la Reine, France

e-mail: bruno.detournay@cemka.fr

S. Dejager · L. Levy-Bachelot · S. Bineau

Laboratoires MSD France, 10-12 Cours Michelet,

92800 Puteaux, France across all care modalities was assessed over the 6-year period.

Results: Ambulatory and hospital care expenditure data for 919 patients with T2D starting DPP4I therapy alone or in combination in 2013 were analyzed. A total of 526 patients $(57.2 \%)$ were still being treated with DPP4I 3 years after DPP4I initiation. Regardless of the treatment regimen, the ambulatory and hospital care costs increased above projected costs in the first year following DPP4I initiation, and then declined during the second and third years to levels in line with or below projected values for patients using DPP4Is as an add-on therapy. The increase in total expenditure in the first year following DPP4I initiation and the subsequent decline in costs in the second and third years were both associated with general trends in consumption across all aspects of patient care.

Conclusion: Despite an initial increase in healthcare expenditure, concomitant with reevaluation of patient care, this study showed that initiation of DPP4Is as an add-on therapy in French patients with T2D was associated with care expenditure that was in line or below predicted values within the 3 years following treatment initiation. Additional studies are required to evaluate the economic impact of the long-term treatment benefits.

Keywords: Budget impact; Claim database; Costs; Diabetes; DPP4I; Time series analysis 


\section{Key Summary Points}

Why carry out this study?

Diabetes is a growing epidemic, with its prevalence in France reaching 5\% of the population in 2015 and resulting in more than 3.3 million people requiring pharmacological treatment.

Type 2 diabetes (T2D) accounts for the vast majority (around 95\%) of these cases, and therefore imposes a substantial economic burden on the French healthcare system.

The aim of the current study was to conduct a longitudinal analysis of realworld data extracted from the EGB (Echantillon Généraliste des Bénéficiaires) database for patients with T2D to determine the total expenditure associated with T2D management before and after the initiation of dipeptidyl peptidase 4 inhibitors (DPP4I).

\section{What was learned from the study?}

Analysis of the data of 919 patients with T2D starting DPP4I therapy alone or in combination in 2013 revealed that ambulatory and hospital care costs increased above projected costs in the first year following DPP4I initiation and that costs then declined during the second and third years to levels in line with or below projected values for patients using DPP4Is as an add-on therapy.

The increase in total expenditure in the first year following DPP4I initiation and the subsequent decline in costs in the second and third years were both associated with general trends in consumption across all aspects of patient care.
The initial increase in healthcare costs in the first year after DPP4I initiation was not linked exclusively to the consequences of initiating DPP4I therapy but rather to expected costs associated with the reevaluation of patient care before introduction of the new treatment regimen and management of the aspects of the disease that led to the decision to introduce DPP4I therapy.

\section{INTRODUCTION}

Diabetes is a growing epidemic that affects quality of life, is associated with significant morbidity, and increases the risk of mortality. It has been widely established that failure to achieve and maintain optimal glycemic control is associated with microvascular and macrovascular complications $[1,2]$. The prevalence of pharmacologically treated diabetes in France has been increasing since 2000 , with average annual growth rates of $5.1 \%$ over the period 2006-2009 and 2.3\% over the period 2009-2013 [3]. Prevalence reached 5\% in 2015, representing more than 3.3 million people in France requiring pharmacological treatment for their diabetes [4]. Type 2 diabetes (T2D) accounts for the vast majority (around 95\%) of these cases, and therefore imposes a substantial economic burden on the French healthcare system $[5,6]$. The overall healthcare expenditure for people with T2D was estimated at $€ 19.5$ billion in 2013, with direct medical costs attributable to T2D and related complications being estimated at $€ 8.5$ billion per year $[6,7]$. The highest individual costs were associated with hospitalizations $(33.2 \%$ of the total cost) and with medication for diabetes, comorbidities, or other related conditions $(23.7 \%$ of the total cost). A significant part of the cost of antidiabetic treatment may be attributed to the use of relatively recent pharmacologic agents in $\mathrm{T} 2 \mathrm{D}$, such as glucagon-like peptide 1 (GLP-1) analogues, dipeptidyl peptidase 4 inhibitors (DPP4Is), and 
new basal insulins. Thiazolidinediones are no longer available in France and sodium/glucose cotransporter 2 (SGLT2) inhibitors are not yet available on the French market.

DPP4Is emerged around 10 years ago as a promising new oral therapy for $\mathrm{T} 2 \mathrm{D}$, leading to significant reductions in hemoglobin A1c (HbA1c) with a low risk of hypoglycemia, weight neutrality, and ease of use $[8,9]$. They are recommended for use in dual therapy or triple therapy with metformin and other glucose-lowering agents, including insulin [10]. They can also be used in monotherapy when metformin is contraindicated or not tolerated [11], although use of DPP4Is alone is not typically reimbursed by the French national healthcare scheme.

Cost-effectiveness and budget impact models are essential components of the economic evaluation into the impact of new treatments on healthcare systems. Many models have been used to report on the cost-effectiveness of DPP4Is for treating T2D $[12,13]$. However, these models are mainly designed to support decisions at market entry. Inputs are based on data obtained from randomized controlled trials (RCTs) and do not take into account the use of DPP4Is in real-world clinical practice [12, 13]. Real-world evidence from sources reflecting the budget holder's perspective, such as data from insurance claims databases, is now being recognized as a valuable resource for economic studies conducted later in product life cycles $[14,15]$. However, without proper adjustment, comparative observational studies using these databases to evaluate the impact of DPP4Is on the cost of T2D treatment are likely to be limited by channeling bias. Baseline characteristics influence disease prognosis and have an impact on treatment costs in diabetes [13], and several studies have shown that patients initiating DPP4I therapy tend to have a poorer health status than those initiating therapy with alternative antihyperglycemic agents [16-19].

Thus, alternative approaches are required to limit channeling bias and allow accurate evaluations of the real-world impact of DPP4Is on the cost of managing T2D. One such approach was recently adopted in a study using data from a French public health insurance database to identify factors associated with cost increases following initiation of insulin therapy [20]. Hanaire et al. used a longitudinal study design, with patients acting as their own controls, to assess the total cost of diabetes care in a cohort of patients in the 3 years preceding initiation of insulin therapy and in the year following insulin initiation [20]. Using this methodology, the aim of the current study was to conduct a longitudinal analysis using an interrupted time series approach to determine the total expenditure associated with T2D management before and after the initiation of DPP4I in a real-world setting.

\section{METHODS}

\section{Study Design}

This retrospective longitudinal study was conducted from a public health insurers' perspective using data on total treatment expenditure extracted from the EGB (Echantillon Généraliste des Bénéficiaires) database for patients with T2D before and after they initiated DPP4I therapy. A schematic representation of the study design is shown in Fig. 1. The index period for initiation of DPP4I therapy was January 2013 to December 2013, with the exact initiation date for each patient being defined as T0. The annual direct total healthcare costs for each patient initiating DPP4I therapy within this index period were then calculated from EGB data for the

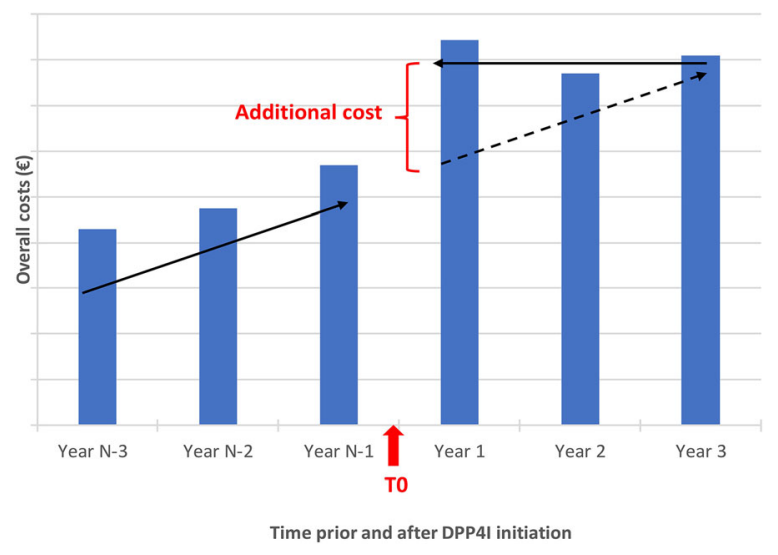

Fig. 1 Study design 
3 years prior to T0 (2010-2012) and 3 years following T0 (2014-2016). Total hospital and ambulatory costs per capita were also calculated. A linear trend was fitted to costs for the 3 years prior to T0 to estimate the projected costs of care over the 3 years after T0. Any additional costs attributable to DPP4I initiation were calculated as the difference between the projected cost and the actual total cost evaluated from EGB data for the 3 years following TO.

\section{Data Source}

The EGB database is a representative and anonymous $1 / 97$ th sample of all beneficiaries of the main French public health insurance scheme. At the time of the study, access to this database was covered through a convention between authors of the study and INSERM, a public scientific and technological institute which operates under the joint authority of the French Ministries of Health and Research. Approval of the study by an ethics committee was deemed unnecessary because all patient data extracted from the EGB database was anonymous.

This national database covers almost $95 \%$ of the French population and the EGB sample contains data for around 600,000 individuals. The EGB contains data on all care consumption eligible for reimbursement, including outpatient and hospital care in both the private and public sectors. Medication, consultations, procedures, paramedical care (nursing, physiotherapy, and paramedics), laboratory tests, medical devices, medical transport, and hospitalizations are all documented for each beneficiary. The presence of a severe chronic disease (Affection de Longue Durée, ALD) is also recorded using international classification of disease (ICD-10) codes. Patients with an ALD are entitled to full insurance coverage for all medical expenses related to the disease. Drugs are recorded using national registration codes (Code Identifiant de Présentation [CIP] and, since 2009, Unité Commune de Dispensation [UCD] codes), and classified using the anatomical therapeutic chemical (ATC) classification system. Hospitalizations are identified by diagnosis-related group (DRG), based on ICD-10 codes. For each item recorded, the date of completion is specified, as well as the date of prescription and the specialty of the prescriber. Over-the-counter medication and treatments not eligible for reimbursement are not documented. Sociodemographic data are limited to age, sex, residence, and date of death.

\section{Identification of Eligible Patients in the EGB Database}

Patients with diabetes were identified in the EGB database using established criteria similar to those described in previous studies $[6,20]$. These criteria were ALD status for diabetes or reimbursement for two or three (depending on pack size) distinct prescriptions for antidiabetic medication in 2013. The decision algorithm described by Charbonnel et al. [6] was then used to distinguish patients with T2D from those with type 1 diabetes. This algorithm was based on the ICD-10 codes (E10 for T1D and E11 for T2D) associated with ALD status or with hospitalizations reported in 2013 with diabetes as the primary or related diagnosis, and on the prescription of insulin.

\section{Study Population and Subgroups}

Eligible participants were aged at least 18 years old with T2D, initiated DPP4I therapy in 2013, and had not received DPP4I treatment in the 3 years prior to initiation. Only patients whose data were available 3 years before (2010-2012) and 3 years after 2013 (2014-2016) were included in the study. Pregnant women were excluded. Data for eligible patients was stratified into three subgroups according to the therapy received: initiation of DPP4I alone; DPP4I therapy combined with one or more oral antidiabetic (OAD) agent(s) without insulin; and DPP4I therapy with insulin, with or without other OAD agents.

\section{Outcomes}

The main study outcome was to determine the total care expenditure for patients with T2D in 
the 3 years before and 3 years after initiation of DPP4I therapy. The evolution of hospital and ambulatory care costs was evaluated during this 6-year period for patients in each of the three treatment regimen subgroups and compared to projected costs. The extent to which individual care modalities contributed to the overall cost of healthcare in these patients was analyzed by studying the distribution of healthcare costs for each subgroup over the 6-year period.

\section{Data Collection}

Total healthcare expenditure included both ambulatory and hospital costs. Ambulatory costs were estimated according to reimbursement data from the EGB database and were composed of expenditure associated with consultations and interventions with physicians and other healthcare professionals (nurses, paramedics, and physiotherapists), pharmaceutical costs, laboratory tests, dentistry, medical devices, ambulatory transport, and other forms of ambulatory care. Public and private hospital costs (including all medical and nonmedical costs) were estimated from the national cost database per DRG. Overall data on cost were retained for each patient for 3 years prior and 3 years following T0.

For each patient, data was also retrieved from the EGB database with regard to age, sex, ALD status, hospitalizations in 2013, and type of physician prescribing DPP4I.

\section{Statistical Analysis}

Quantitative data were presented as mean $\pm s$ tandard deviation (SD) or median values, and qualitative data as frequency counts and percentages. Appropriate statistical tests were used to assess cross-sectional analyses: the chisquared test or Fisher's exact test for qualitative variables, Student's $t$ test for quantitative variables, and the Wilcoxon test for paired samples. All tests were interpreted with an alpha risk of $5 \%$.

All statistical analyses were conducted using SAS software, version 9.2 (Cary, NC, USA).

\section{RESULTS}

\section{Study Population}

A total of 414,800 patients aged at least 18 years old were identified in the EGB database in 2013. Of these, 21,815 were identified as having T2D based on ICD-10 codes used at hospitalization for diabetes or for ALD status, and 919 initiated DPP4I therapy during the index period (Fig. 2).

\section{Clinical and Demographic Data}

Patient demographics and clinical data at the time of initiation of DPP4I therapy are shown in Table 1. DPP4I combined with one or more OAD agent(s) was the most common form of therapy $(n=709 / 919,77.1 \%)$. Only $8.6 \%$ of patients $(n=79 / 919)$ initiated DPP4I treatment as a monotherapy and $14.3 \%$ of patients

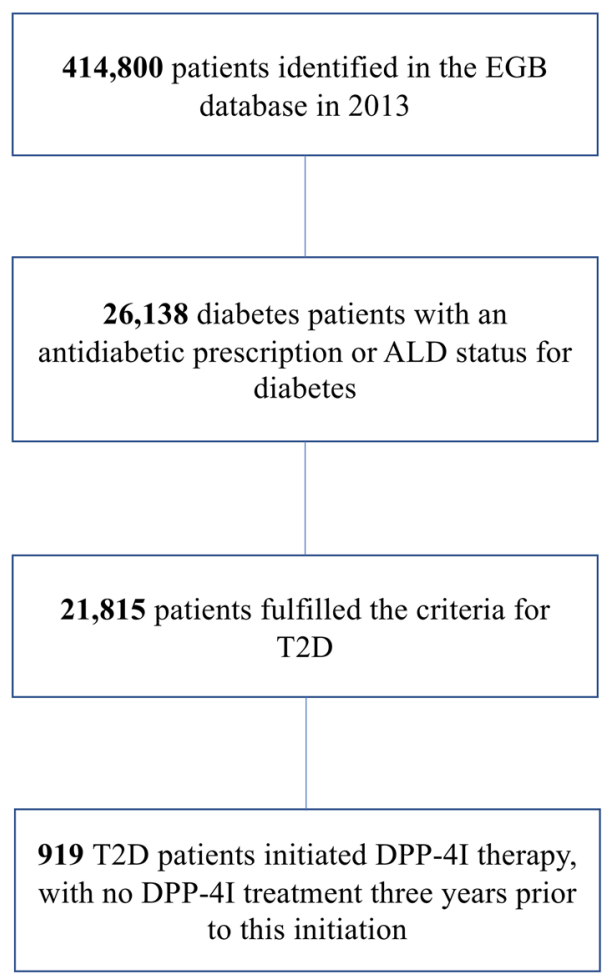

Fig. 2 Flow chart of patients with diabetes in the EGB (Echantillon Généraliste des Bénéficiaires) database. ALD affection de longue durée, T2D type 2 diabetes, DPP4I dipeptidyl peptidase 4 inhibitor 
Table 1 Patient demographics, care characteristics, and therapeutic regimens around the time of initiation of DPP4I therapy

Patient demographics and care characteristics

$N=919$

Gender, $n(\%)$

Male

Age, years

Mean (SD)

$63.2(12.4)$

Range (min-max)

ALD status for diabetes, $n(\%)$

Yes

Hospitalization in $2013^{\mathrm{a}}, n$ (\%)

Yes

Therapeutic regimens, $n$ (\%)

DPP4I alone

DPP4I with one or more OAD agent

Metformin and DPP4I

$355(38.6)$

SUs and DPP4I

$69(7.5)$

One other OAD and DPP4I

Triple therapy with metformin, SUs, and DPP4I

$>1$ other OAD and DPP4I

$45(4.9)$

Other multiple therapy: OAD and DPP4I

DPP4I with insulin and one or more OAD agent

Insulin, metformin, and DPP4I

$39(4.2)$

Insulin, metformin, SUs, and DPP4I

$31(3.4)$

Other insulin therapy, other OAD, and DPP4I

$61(6.6)$

Data presented were collected for the first 3 months following initiation of DPP4I therapy

$A L D$ Affection de Longue Durée (severe chronic disease), DPP4I dipeptidyl peptidase 4 inhibitor, $O A D$ oral antidiabetic, $S U$ s sulfonylureas, $S D$ standard deviation, $N$ number of patients

${ }^{a}$ Includes all hospitalizations, including overnight stays, stays of $<24 \mathrm{~h}$ and scheduled visits

$(n=131 / 919)$ started DPP4I therapy in combination with insulin, with or without an OAD (Table 1). Sitagliptin was the most prescribed type of DPP4I (around 60\% of patients), either with $(26 \%$ of patients; $n=239 / 919)$ or without (36.9\% of patients; $n=339 / 919$ ) metformin (chi-square test, $p<0.0001$ ). Vildagliptin and saxagliptin were prescribed in combination with metformin in $12 \%(n=110 / 919)$ and $5.8 \%$ $(n=53 / 919)$ of patients, and alone in $10.8 \%$ $(n=99 / 919)$ and $8.7 \%(n=80 / 919)$ of patients, respectively.

Overall, DPP4I therapy was most commonly prescribed by general practitioners $(n=656$, $71.4 \%)$ and only $18.8 \%$ of patients $(n=173)$ had their DPP4I prescription initiated by a hospital physician, $7.6 \% \quad(n=70)$ by an endocrinologist, and $2.2 \%(n=20)$ by another 
specialist. Although the overall rate of initiation during a hospitalization was $18.8 \%$, the rate was much higher $(43.5 \%)$ when DPP4I therapy was initiated in combination with insulin $(n=57 /$ 131) (Fisher's exact test, $p<0.0001$ ).

\section{Therapeutic Regimens in the 3 Months Preceding DPP4I initiation}

In the 3 months prior to DPP4I initiation, the majority of patients were treated with only one OAD $(n=427 / 919 ; 46.5 \%)$, most commonly metformin $(n=340 ; 37 \%)$. Treatment with insulin, with or without therapy with an OAD was reported in $11.6 \%$ of patients $(n=107)$. A total of $16.4 \%$ of patients $(n=151)$ had not been treated with any OAD in the 3 months before initiating DPP4I therapy.

\section{Rates of Discontinuation of DPP4I Therapy and Switch to Insulin Therapy in the 3 Years Following DPP4I Initiation}

Less than a third of patients discontinued DPP4I therapy in the first year after initiation $(28.7 \%$, $n=264)$. In the last quarter of the third year after DPP4I initiation, $57.2 \%$ of patients were still continuing their treatment. The main group of patients, i.e., those initiating DPP4I therapy in combination with OADs without insulin $(n=709)$, were also the most likely to have remained on DPP4I therapy over the 3 -year period ( $n=427,60.2 \%)$, followed by the small group of patients initiating DPP4Is as a monotherapy $(n=45 / 79,57.0 \%)$. Patients initiating DPP4I therapy in combination with insulin $(n=131)$ were the least likely to maintain DPP4I therapy over the 3-year period $(n=54,41.2 \%)$.

Switch to insulin 3 years following DPP4I initiation was observed in $11.4 \%$ of patients $(n=9 / 79)$ treated with DPP4I alone and in $14.5 \%$ of patients $(n=103 / 709)$ initiating DPP4I therapy in combination with another OAD $(p<0.0001)$.

\section{Evolution of Ambulatory and Hospitalization Costs Before and After Initiation of DPP4I Therapy According to Treatment Regimen}

Total ambulatory and hospital care costs for the 3 years prior and 3 years following initiation of DPP4I therapy are shown in Fig. 3. Hospital and ambulatory costs per capita are shown in Fig. S1 in the supplementary material. For all three treatment groups, the combined cost of ambulatory and hospital care showed a marked increase above projected values in the first year following DPP4I initiation. The increase in total costs at 1 year was mainly driven by a $125 \%$ rise in hospital costs for patients in the DPP4I monotherapy subgroup (Fig. 3a). The cost increases in the first year after DPP4I initiation for patients receiving DPP4I in combination with another OAD without insulin and those initiating DPP4I therapy combined with insulin were driven by a $40 \%$ increase in ambulatory costs in both groups, and by a $56 \%$ increase in hospital costs for the DPP4I in combination with another OAD without insulin group and a $19.7 \%$ increase in hospital costs in the DPP4I therapy combined with insulin group (Fig. 3b, c).

Expenditure during the second and third years following DPP4I initiation declined, relative to the 1-year peak in expenditure, for all patient subgroups (Fig. 3). For patients in the DPP4I monotherapy group $(n=79)$, total costs fell during the 3 years after initiation relative to the 1-year peak in expenditure, but remained slightly above the projected care costs. This additional cost was mainly driven by hospital care consumption (Fig. 3a). In the main group of patients initiating DPP4I therapy in combination with another OAD without insulin $(n=709)$, no additional care costs after 3 years were reported, with both hospital and ambulatory care consumption falling over the second and third years to levels in line with or below projected costs (Fig. 3b). A large fall in total care costs to well below the projected level was observed by 3 years following DPP4I initiation in patients initiating the therapy in combination with insulin $(n=131)$ (Fig. 3c). The large decrease in costs for this patient group was 

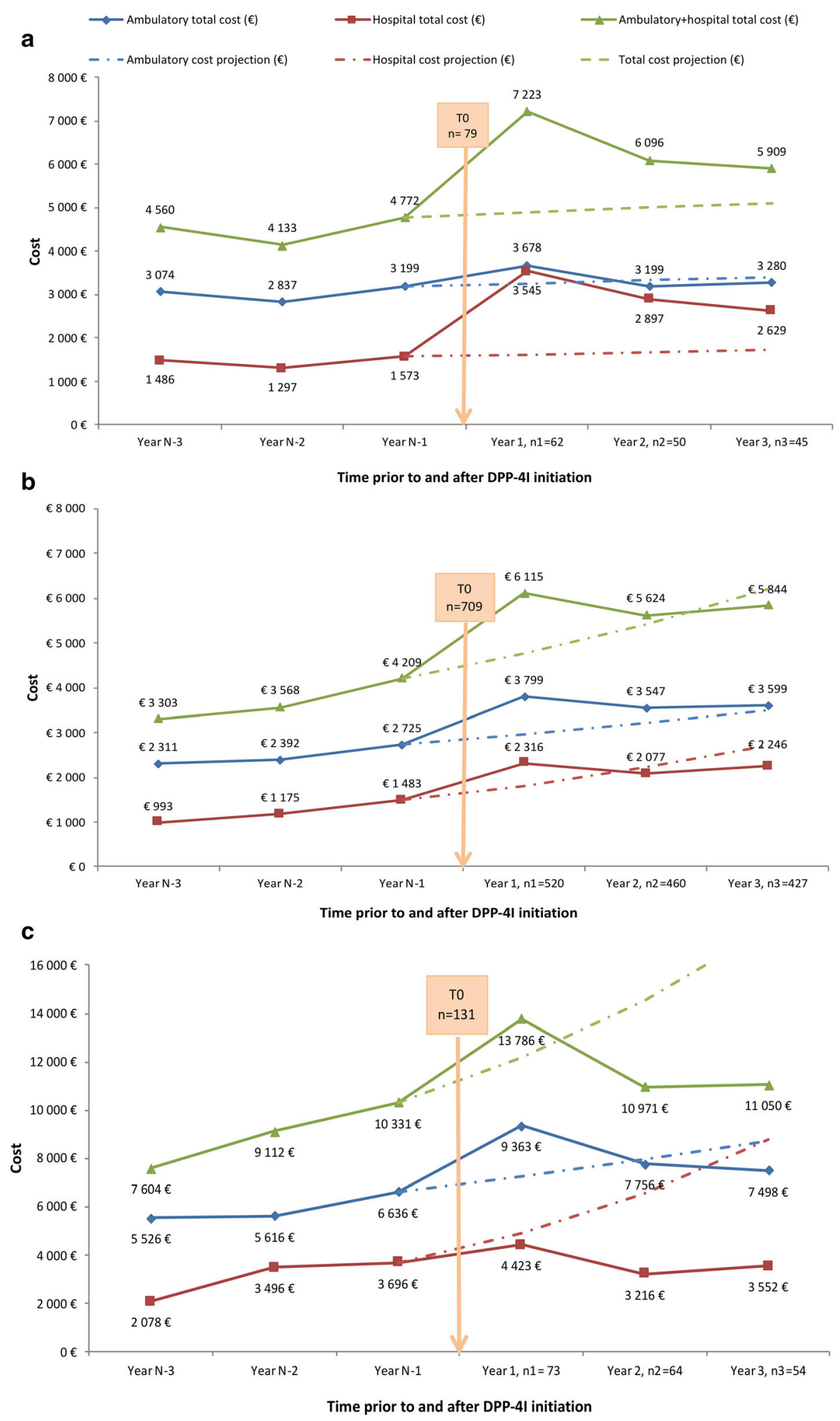
4Fig. 3 Evolution of total healthcare expenditure during the 3 years before and after initiation of DPP4I therapy in a the DPP4I monotherapy group $(n=79)$, b the DPP4I combined with another OAD without insulin group $(n=709)$, and $\mathbf{c}$ the DPP4I combined with insulin $(n=131)$ group. DPP4I dipeptidyl peptidase 4 inhibitor, OAD oral antidiabetic, n1-n3 number of patients who continued DPP4I therapy 1-3 years after initiation

driven by a large fall in costs associated with hospitalization of these patients (Fig. 3c).

\section{Distribution of Care Costs Before and After Initiation of DPP4I Therapy According to Treatment Regimen}

The distribution of costs across the main ambulatory care modalities for the three patient subgroups over the 3 years before and 3 years following DPP4I initiation are shown in Fig. 4.

The increase in expenditure in the first year following DPP4I initiation was associated with a general trend of increased consumption across all aspects of ambulatory care, regardless of the treatment regimen being used. Although the main contributor to the overall rise in care costs for patients in the DPP4I monotherapy group was the large rise in hospital costs (Fig. 3), a 40\% rise in pharmaceutical costs-associated with the cost of DPP4I and all other agents-was also observed in this subgroup of patients (Fig. 4a). For patients treated with DPP4I combined with another OAD without insulin, the cost increase for ambulatory care in the first year after DPP4I initiation was associated with a $65 \%$ increase in costs for all pharmaceuticals and a 96\% increase in costs associated with other healthcare professionals (Fig. 4b). Similarly, a $61 \%$ increase in costs for all pharmaceuticals was observed in the first year after initiation in patients receiving DPP4I in combination with insulin, whereas costs associated with other healthcare professionals appeared stable during this period (Fig. 4c).

The continued relative decline in healthcare costs during the second and third years following initiation of DPP4I treatment was observed across all subgroups and was also associated with a trend towards reduced consumption across all aspects of ambulatory care. Decreases in expenditure relative to the 1 -year peak in costs were observed for costs associated with all pharmaceuticals in all three patient groups: $19 \%, 21 \%$, and $29 \%$ decreases in pharmaceutical costs were observed by the third year for the DPP4I monotherapy, DPP4I combined with another OAD without insulin, and DPP4I combined with insulin subgroups, respectively (Fig. 4a-c).

\section{DISCUSSION}

This longitudinal study used data from a representative sample of the French national health insurance claims database to provide real-world evidence of the costs associated with the initiation of DPP4I among patients with T2D.

Major advances and rapid growth of the noninsulin antidiabetic drug market have been observed over the past decade, primarily driven by the introduction of new classes of drugs, such as DPP4Is, SGLT2 inhibitors, and GLP-1 analogues [21]. SGLT2 inhibitors are not currently marketed in France, but in 2017 the DPP4I and GLP-1 analogue drug classes accounted for $43 \%$ and $29 \%$, respectively, of the total cost of sales of noninsulin antihyperglycemic agents available on the market and reimbursed in France [22]. The price of such innovative new drugs is always a matter of debate, and it is difficult to obtain a clear view of the true price of these treatments after offinvoice price rebates and allowances, managed entry agreements, and patient access schemes have been considered.

A systematic review of 11 cost-effectiveness studies concluded that DPP4Is were likely to represent a cost-effective option, as compared to sulfonylureas (SUs) and insulin, when used as an add-on treatment in patients with T2D who had not achieved glycemic targets with antidiabetic monotherapy [12]. However, heterogeneity in the methodology and data sources used by the medico-economic evaluations included in the review prevented the authors from conducting a meta-analysis of the results [12]. Similar conclusions on the cost- 

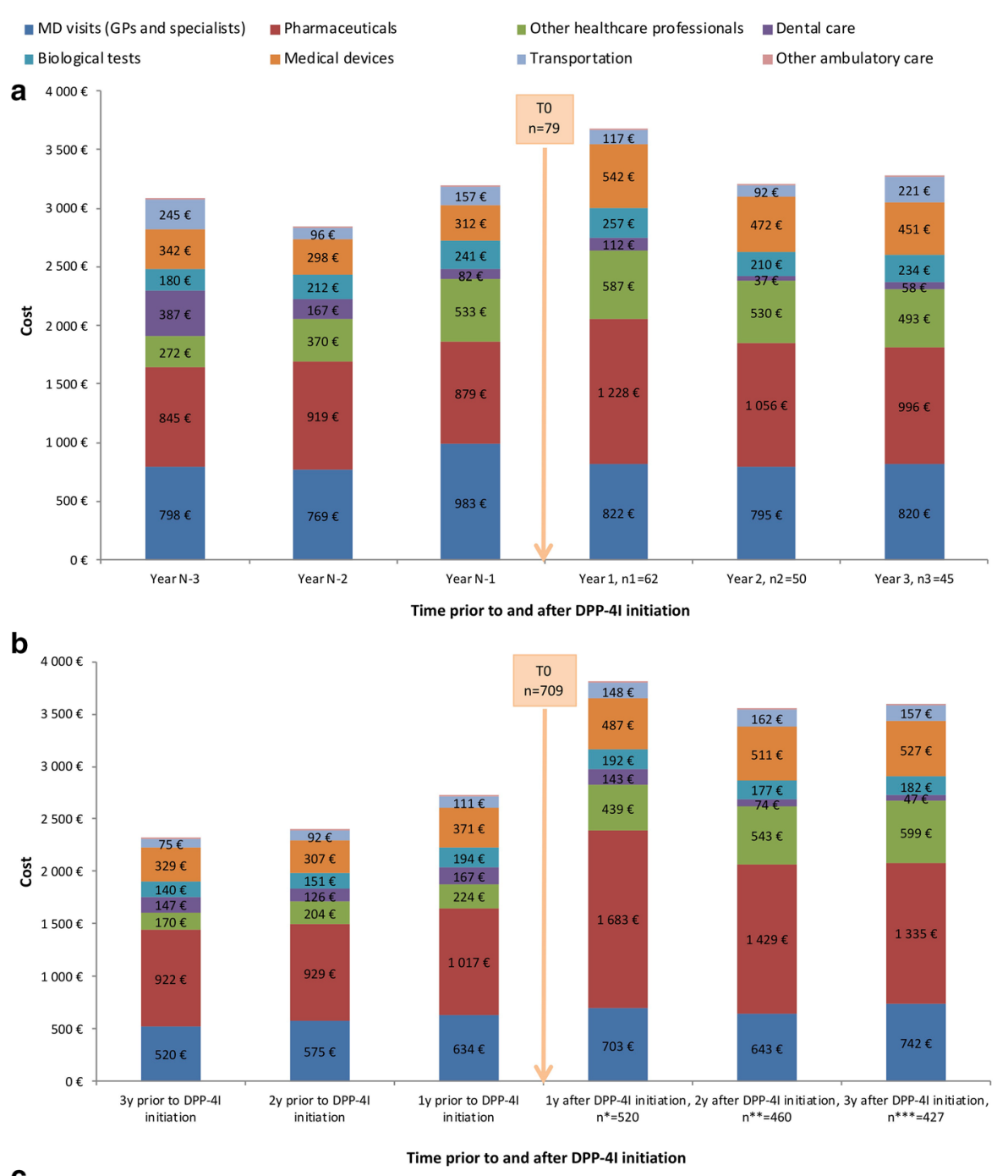

C

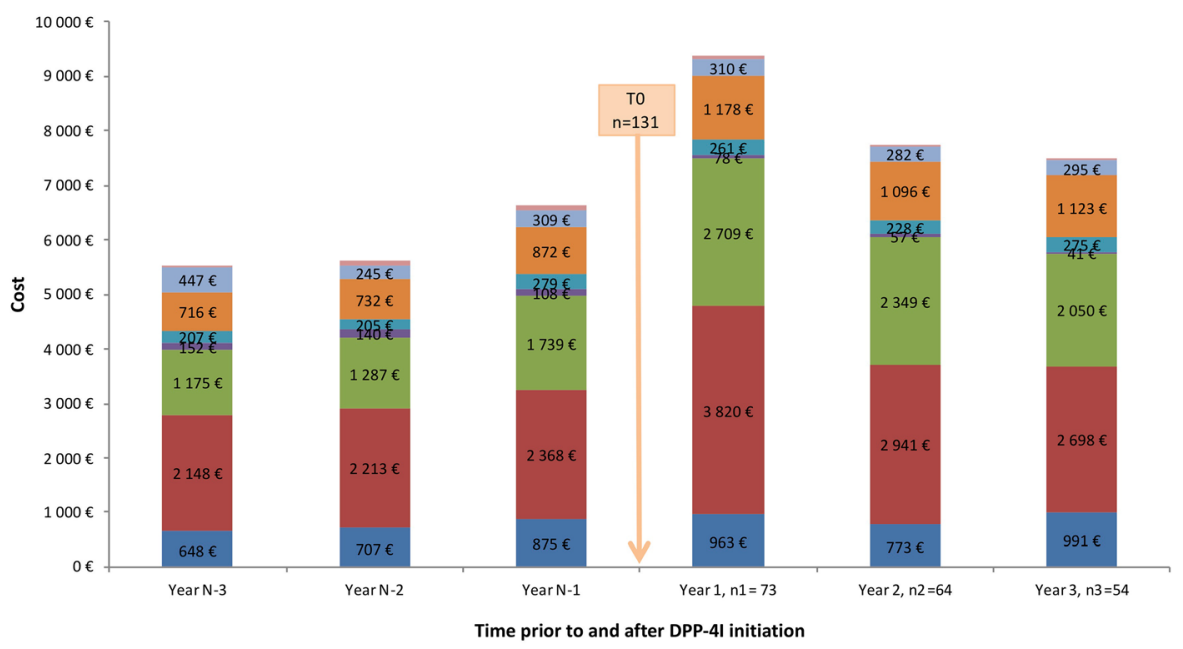


4Fig. 4 Distribution of expenditure across healthcare modalities during the 3 years before and after initiation of DPP4I therapy in a the DPP4I monotherapy group $(n=79)$, b the DPP4I combined with another OAD without insulin group $(n=709)$, and $\mathbf{c}$ the DPP4I combined with insulin $(n=131)$ group. DPP4I dipeptidyl peptidase 4 inhibitor, OAD oral antidiabetic, y year(s), n1-n3 number of patients who continued DPP4I therapy 1-3 years after initiation. Other ambulatory care covers all other costs, such as spa care, partially or totally reimbursed by the French national healthcare scheme

effectiveness of DPP4Is were drawn in a more recent systematic review conducted by Baptista et al. [13]. These authors also commented on the fact that most of the DPP4I cost-effectiveness models used so far had been conducted using data from a limited number of RCTs, which may not be truly representative of the use of DPP4Is in real-world practice [13].

Our approach was based on a pre-post comparison of total costs of diabetes care around the time of DPP4I initiation using data obtained from a real-world source. The data used in this study can be considered as representative of the general population of patients with T2D as it was obtained from a database constructed from a random sample of exhaustive data on healthcare reimbursement for the general French population. Our analysis revealed a marked increase in ambulatory and hospital care costs above projected values at the time of initiation of DPP4I therapy, regardless of the treatment regimen prescribed. Evaluation of the distribution of consumption showed that higher levels of expenditure across all care modalities contributed to the increase in costs in the first year following DPP4I initiation. Thus, the increase in cost associated with initiation of DPP4I therapy in these patients was not solely attributed to an increase in pharmaceutical costs for DPP4I and all other agents, or to factors specific to initiation of DPP4I treatment, but resulted from a culmination of additional expenditure. The longitudinal study conducted by Hanaire et al. [20], evaluating the cost associated with initiation of insulin therapy, led the authors to draw a similar conclusion: increased costs around the initiation of insulin therapy could not be exclusively attributed to the consequences of insulin prescription. In the case of DPP4I therapy, the increased pharmaceutical costs observed in the first year after DPP4I initiation also included the costs associated with all other treatments, such as those required for the management of associated cardiovascular risk factors. Expenditure across all healthcare modalities around the time of DPP4I initiation would have been dependent on the health status of the patient just before and after initiation of DPP4I therapy. Thus, reevaluation of the severity of disease before treatment escalation or interventions to treat the factors (worsening of diabetes, comorbidities, or the occurrence of complications) that led to initiation of DPP4I treatment would have led to the increase in healthcare consumption at around the time of DPP4I initiation.

For all patient subgroups, the initial increase in costs was followed by a continued decline in ambulatory and hospital care costs over the second and third years following DPP4I initiation. Declining costs across all sectors led consumption to fall in line with or below projected values within 3 years following DPP4I initiation for patients where DPP4I was used as an add-on therapy with insulin or other OADs. The decline in costs over the second and third years following DPP4I initiation appears to be linked to stabilization of patient health status in response to DPP4I therapy and the other care strategies implemented over the period analyzed. Indeed, numerous studies have demonstrated the effectiveness and safety of DPP4I treatment, both as a monotherapy and in combination with other oral antidiabetic agents and insulin $[9,23,24]$. Further evaluations over the longer term would be required to determine if costs fell further in response to the benefits of DPP4I therapy on glycemic control.

One of the strengths of our study was that the longitudinal design minimized the impact of confounding and channeling bias. Several studies have shown that patients with T2D prescribed the DPP4I sitagliptin tended to be older, had more diabetes complications and comorbidities, and had greater use of prescription medications and more physician visits than patients initiating treatment with other oral 
antihyperglycemic agents [16-19]. The longitudinal design used in our study allowed comparisons of treatment costs before and after DPP4I initiation to be conducted with patients acting as their own controls, thus limiting the impact of channeling bias on the results of our analysis. Another strength of the study was that longitudinal analysis of the total expenditure associated with management of $\mathrm{T} 2 \mathrm{D}$ around the time of DPP4I initiation allowed the costs associated with a range of care modalities to be evaluated and major contributors to the increase in healthcare costs to be identified. This type of information is essential for the implementation of effective strategies to reduce the costs associated with the initiation of new treatments.

Although our results provide an interesting view of the cost consequences of prescribing a DPP4I for management of T2D in a real-life setting, our study had several limitations. One limitation was that this study used an interrupted time series design to analyze the economic effects of initiating DPP4I therapy. Although this type of design is widely used in studies of public health interventions, this approach did not allow confounding due to other interventions or events occurring around the time of DPP4I initiation-which may also have affected the economic outcome-to be excluded [25]. However, given that the period around DPP4I initiation analyzed in our study was relatively short, it is unlikely that there would have been any major changes in other factors that could have accounted for the observed trends. Another limitation was that the cost evaluation was performed using an intention to treat analysis, using data for all patients eligible for the study at the time of DPP4I initiation, including patients who discontinued DPP4I therapy before the end of the 3 -year post-initiation evaluation period. A previous real-life observational study comparing treatment maintenance in patients with T2D receiving dual therapy with sitagliptin and metformin with that of patients receiving metformin and SUs found that the median maintenance period for the DPP4I dual therapy was 43.2 months with discontinuation rates over the 3-year follow-up period of $33.1 \%$, whereas that for the dual therapy with metformin and
SUs was 20.2 months, with discontinuation rates of $46.5 \%$ [26]. A slightly higher discontinuation rate of $39.8 \%$ after 3 years was observed in our main subgroup of patients initiating DPP4I therapy in combination with another OAD without insulin, and a discontinuation rate of $42.8 \%$ after 3 years was observed for the whole population. Although we did not analyze the impact of DPP4I therapy discontinuation on the economic outcome in our study, discontinuation of a diabetes therapy would generally be expected to result in an increase in healthcare costs due to switching to potentially more expensive alternative treatments. Although the EGB is an exhaustive and representative sample of the French population, the database itself imposed some study limitations: diagnoses were only recorded for hospitalized patients or those with ALD status; there may have been errors in the coding of some treatments and interventions; patients were identified using algorithms which could have led to errors in some cases; some patient subgroups-such as students and civil servants—are poorly represented in the database; data is only available for prescribed treatments and not for treatments actually delivered; and, finally, only limited demographic and clinical data are reported for patients included in the database.

\section{CONCLUSION}

Our real-world longitudinal study revealed that, despite an initial increase in ambulatory and hospital costs, initiation of add-on DPP4I therapy in patients with T2D in France was associated with care expenditure that was in line with or below predicted values within the 3 years following treatment initiation. The initial increase in healthcare costs in the first year after DPP4I initiation was associated with increased expenditure across the range of care modalities used to manage the patients with T2D and was not linked exclusively to the consequences of initiating DPP4I therapy. The reevaluation of patient care before introduction of the new treatment regimen and management of the aspects of the disease that led to the decision to introduce DPP4I therapy would be expected to 
result in short-term increases in healthcare expenditure around the time of treatment initiation. Increasing our understanding of the factors contributing to the totality of costs associated with treatment evaluation and the decision to introduce a new treatment is essential for decision-makers. Finally, our study highlights the importance of evaluating all aspects of patient care in both the longer and shorter term when conducting budget impact analyses after the initiation of a new therapy.

\section{ACKNOWLEDGEMENTS}

Funding. This study and the Rapid Service Fee were sponsored by Merck, Sharp and Dohme (MSD) France.

Medical Writing. We thank Emma Pilling and Marielle Romet (Santé Active Edition/Synergy Pharm) for medical writing assistance funded by MSD France.

Authorship. All named authors meet the International Committee of Medical Journal Editors (ICMJE) criteria for authorship for this article, take responsibility for the integrity of the work as a whole, and have given their approval for this version to be published.

Authorship Contributions. LLB, SD, BD were involved in the conception and design of the study. CE and ET performed the statistical analysis. $\mathrm{BD}, \mathrm{SB}, \mathrm{CE}$ contributed to the interpretation of the data. $\mathrm{BD}$ and SD drafted the paper and revised the paper critically for intellectual content.

Disclosures. Corinne Emery, Elodie Torreton and Bruno Detournay are employed by CEMKA, a consulting team specializing in health economics, epidemiology, and outcomes research. Bruno Detournay also received personal compensation for board participation and speaking fees from MSD, Novo-Nordisk, Sanofi, Lilly and Pfizer. Sylvie Dejager, Laurie LevyBachelot and Sébastien Bineau are employed by MSD France.
Compliance with Ethics Guidelines. Approval of the study by an ethics committee was deemed unnecessary because all patient data extracted from the EGB database was anonymous.

Data Availability. Access to EGB data is now strictly regulated by law and therefore the data will not be deposited.

Open Access. This article is distributed under the terms of the Creative Commons Attribution-NonCommercial 4.0 International License (http://creativecommons.org/licenses/ by-nc/4.0/), which permits any noncommercial use, distribution, and reproduction in any medium, provided you give appropriate credit to the original author(s) and the source, provide a link to the Creative Commons license, and indicate if changes were made.

\section{REFERENCES}

1. Khunti K, Seidu S. Therapeutic inertia and the legacy of dysglycemia on the microvascular and macrovascular complications of diabetes. Diabetes Care. 2019;42(3):349.

2. Laiteerapong N, Ham SA, Gao Y, et al. The legacy effect in type 2 diabetes: impact of early glycemic control on future complications (the Diabetes and Aging Study). Diabetes Care. 2019;42(3):416-26.

3. Regnault N, Mandereau-Bruno L, Denis P, FagotCampagna A, Fosse-Edorh S. O31 Évolution de la prévalence du diabète traité pharmacologiquement, France, 2006-2013; 2015.

4. Mandereau Bruno L, Fosse Edorh S. Prévalence du diabète traité pharmacologiquement (tous types) en France en 2015. Disparités territoriales et socioéconomiques. Bull Epidémiol Hebd. 2017;2017(2728):586-91.

5. Druet C, Roudier C, Romon I, Assogba FGA, Bourdel Marchasson I, Eschwege E, Risse Fleury M, Poutignat N, Gautier A, Chantry M, Weill A, Fosse S, Fagot Campagna A. Echantillon national témoin représentatif des personnes diabétiques, Entred 2007-2010. Caractéristiques, état de santé, prise en charge et poids économique des personnes diabétiques. Santé Publique France Official Report. 2012. Available on: https://www.santepubliquefrance.fr/ maladies-et-traumatismes/diabete/documents/ 
rapport-synthese/echantillon-national-temoin-repr esentatif-des-personnes-diabetiques-entred-20072010.-caracteristiques-etat-de-sante-prise-en-charge -et-poids-ec. Accessed 12 Jan 2020.

6. Charbonnel B, Simon D, Dallongeville J, et al. Direct medical costs of type 2 diabetes in France: an insurance claims database analysis. PharmacoEcon Open. 2017;2(2):209-19.

7. de Lagasnerie G, Aguadé A-S, Denis P, Fagot-Campagna A, Gastaldi-Menager C. The economic burden of diabetes to French national health insurance: a new cost-of-illness method based on a combined medicalized and incremental approach. Eur J Health Econ. 2018;19(2):189-201.

8. Amori RE, Lau J, Pittas AG. Efficacy and safety of incretin therapy in type 2 diabetes systematic review and meta-analysis. JAMA. 2007;298(2): 194-206.

9. Gao W, Dong J, Liu J, et al. Efficacy and safety of initial combination of DPP-IV inhibitors and metformin versus metformin monotherapy in type 2 diabetes: a systematic review of randomized controlled trials. Diabetes Obes Metab. 2014;16(2): 179-85.

10. Inzucchi SE, Bergenstal RM, Buse JB, et al. Management of hyperglycaemia in type 2 diabetes, 2015: a patient-centred approach. Update to a position statement of the American Diabetes Association and the European Association for the Study of Diabetes. Diabetologia. 2015;58(3):429-42.

11. Gallwitz B. Management of patients with type 2 diabetes and mild/moderate renal impairment: profile of linagliptin. Ther Clin Risk Manag. 2015;11:799-805.

12. Geng J, Yu H, Mao Y, Zhang P, Chen Y. Cost effectiveness of dipeptidyl peptidase-4 inhibitors for type 2 diabetes. PharmacoEconomics. 2015;33(6):581-97.

13. Baptista A, Teixeira I, Romano S, Carneiro AV, Perelman J. The place of DPP-4 inhibitors in the treatment algorithm of diabetes type 2: a systematic review of cost-effectiveness studies. Eur J Health Econ. 2017;18(8):937-65.

14. Bowrin K, Briere J-B, Levy P, Millier A, Clay E, Toumi M. Cost-effectiveness analyses using realworld data: an overview of the literature. J Med Econ. 2019;20:1-9.

15. Sullivan SD, Mauskopf JA, Augustovski F, et al. Budget impact analysis-principles of good practice: report of the ISPOR 2012 Budget Impact Analysis Good Practice II Task Force. Value Health. 2014;17(1):5-14.
16. Wang T, McNeill AM, Chen Y, O’Neill EA, Engel SS. Characteristics of elderly patients initiating sitagliptin or non-DPP-4-inhibitor oral antihyperglycemic agents: analysis of a cross-sectional US claims database. Diabetes Ther. 2018;9(1):309-15.

17. Zhang Q, Rajagopalan S, Mavros P, et al. Baseline characteristic differences between patients prescribed sitagliptin vs. other oral antihyperglycemic agents: analysis of a US electronic medical record database. Curr Med Res Opin. 2010;26(7):1697-703.

18. Brodovicz KG, Chen Y, Liu Z, Ritchey ME, Liao J, Engel SS. Characterization of sitagliptin use in patients with type 2 diabetes and chronic kidney disease by crosssectional analysis of a medical insurance claims database. Diabetes Ther. 2015;6(4):627-34.

19. Cai B, Katz L, Alexander CM, Williams-Herman D, Girman CJ. Characteristics of patients prescribed sitagliptin and other oral antihyperglycaemic agents in a large US claims database. Int J Clin Pract. 2010;64(12):1601-8.

20. Hanaire H, Attali C, Lecointre B, et al. Déterminants des coûts du passage à l'insuline en France chez le patient diabétique de type 2: quelles pistes d'optimisation? Santé Publ. 2016;28(6):781-9.

21. Bosnic N, Zhang Y, O'Shea B, Lungu E. The cost of new oral anti-diabetic drugs in Canada and internationally. Value Health. 2018;21:S81.

22. Caisse Nationale de l'Assurance Maladie. Données mensuelles sur les médicaments délivrés par les pharmacies de ville et remboursés par l'Assurance Maladie; 2017. https://www.ameli.fr/l-assurancemaladie/statistiques-et-publications/donnees-statis tiques/medicament/medicamentspharmacies-de-villepar-classe-atc/medic-am-labellise-2017.php. Accessed 12 Jan 2020.

23. Scheen A. DPP-4 inhibitors in the management of type 2 diabetes: a critical review of head-to-head trials. Diabetes Metab. 2012;38(2):89-101.

24. Karagiannis T, Paschos P, Paletas K, Matthews DR, Tsapas A. Dipeptidyl peptidase- 4 inhibitors for treatment of type 2 diabetes mellitus in the clinical setting: systematic review and meta-analysis. BMJ. 2012;344:e1369.

25. Lopez Bernal J, Cummins S, Gasparrini A. The use of controls in interrupted time series studies of public health interventions. Int J Epidemiol. 2018;47(6): 2082-93.

26. Valensi P, de Pouvourville G, Benard $\mathrm{N}$, et al. Treatment maintenance duration of dual therapy with metformin and sitagliptin in type 2 diabetes: the ODYSSEE observational study. Diabetes Metab. 2015;41(3):231-8. 